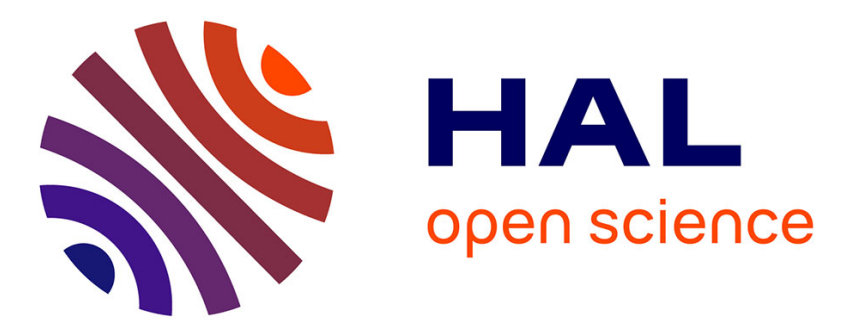

\title{
Probability distribution function based iris recognition boosted by the mean rule
}

Kert Pjatkin, Morteza Daneshmand, Pejman Rasti, Gholamreza Anbarjafari

\section{To cite this version:}

Kert Pjatkin, Morteza Daneshmand, Pejman Rasti, Gholamreza Anbarjafari. Probability distribution function based iris recognition boosted by the mean rule. 2014 International Conference on Intelligent Computing and Internet of Things (ICIT), Jan 2015, Harbin, China. pp.47-50, 10.1109/ICAIOT.2015.7111535 . hal-02527965

\section{HAL Id: hal-02527965 \\ https://univ-angers.hal.science/hal-02527965}

Submitted on 2 Sep 2021

HAL is a multi-disciplinary open access archive for the deposit and dissemination of scientific research documents, whether they are published or not. The documents may come from teaching and research institutions in France or abroad, or from public or private research centers.
L'archive ouverte pluridisciplinaire HAL, est destinée au dépôt et à la diffusion de documents scientifiques de niveau recherche, publiés ou non, émanant des établissements d'enseignement et de recherche français ou étrangers, des laboratoires publics ou privés. 


\title{
Probability Distribution Function based Iris Recognition Boosted by The Mean Rule
}

\author{
Kert Pjatkin, Morteza Daneshmand, Pejman Rasti, and Gholamreza Anbarjafari* \\ iCV Group, IMS Lab, Institute of Technology, \\ University of Tartu, Nooruse 1, Tartu 50411, Estonia \\ *sjafari@ut.ee
}

\begin{abstract}
In this work, a new iris recognition algorithm based on tonal distribution of iris images is introduced. During the process of identification probability distribution functions of colored irises are generated in HSI and YCbCr color spaces. The discrimination between classes is obtained by using KullbackLeibler divergence. In order to obtain the final decision on recognition, the multi decision on various color channels has been combined by employing mean rule. The decisions of $\mathrm{H}, \mathrm{S}, \mathrm{Y}, \mathrm{Cb}$ and $\mathrm{Cr}$ color channels have been combined. The proposed technique overcome the conventional principle component analysis technique and achieved a recognition rate of $100 \%$ using the UPOL database. The major advantage is the fact that it is computationally less complex than the Daugman's algorithm and it is suitable for using visible light camera as opposed to the one proposed by Daugman where NIR cameras are used for obtaining the irises.
\end{abstract}

Keywords-Probability distribution function; Kullback-Leibler divergence; Iris recognition;Classification; Mean rule

\section{INTRODUCTION}

Several ophthalmologists notice during clinical research that the patterns on the iris are clearly distinctive and have unique texture potentially used successfully as a mean of biometric identification and it doesn't change during the lifespan of a person [1,2]. J. G. Daugman took advantage of the previous ideas and introduced the first and still the most widely used algorithm for iris recognition in 1993 [1].

Daugman explains that the human iris has at least several hundred degrees of freedom [1]. Being more precise, in a later research in cooperation with $\mathrm{C}$. Downing, they discovered by examining 2.3 million iris pairs that an iris has 244 independent degrees of freedom [3]. Therefore, iris recognition is considered to be one of the most foolproof biometric person recognition methods nowadays and it is regarded as the most reliable mean of biometric identification out of the seven most popular ones [4].

The morphogenesis of an iris is random and the physical appearance depends heavily on the conditions during the fetus. Even identical twins do not have the same patterns and color. What is more, iris is counted as the only internal organ visible outside and hence, it is practically impossible to shape the pattern of it [5].

The current algorithm dominating the market is the one created and patented by Daugman. The method uses an integrodifferential operator that is described by eqn.(1). Eqn. (1) is used twice during the first iteration looking for the outer boundaries of an iris and during the second run removing the pupil from the previously found iris. After obtaining the real iris, the next step is to transfer the pixels from traditional Cartesian coordinate space to the polar coordinate system in order to maintain the reference to the same iris points independent on the angel under which the photo was. During the pattern analysis the upper part and 45 degree slice of the lower part of the iris are excluded, because these are the regions where most probably a disturbing noise, like eyelids or reflections, exists [1].

$$
\max _{\left(r, x_{0}, y_{0}\right)}=\left|G_{0}(r) * \frac{\partial}{\partial r} \oint_{r, x_{0}, y_{0}} \frac{I(x, y)}{2 \pi r} d s\right|
$$

$G_{0}(r)$ is Gaussian blur, * is convolution, $I(x, y)$ is location to apply the circle, and $r$ is circle radius. The algorithm used Gabor filter to generate numerical comparable data out of the filtered images and used the Hamming window to compare two irises [1].

The most significant difference of our proposed algorithm to the one that Daugman created is that the proposed method is taking advantage of the color information of the irises, whereas Daugman uses just the patterns to detect irises and all the images have to be taken with IR cameras. The latter are mostly used because the image reveals usually more of the texture of an iris than using a visible light camera [6], but the traditional color cameras are more widespread today and therefore a suitable algorithm using color information would make the recognition process more robust [7].

Anbarjafari and Demirel have proposed a similar statistical algorithm for iris recognition that uses tonality information of the irises. In their method they have represented an iris image by sets of its histogram in HSI color space. Then they have used cross correlation in order to obtain the recognition in each class. Furthermore majority voting has been employed to combine the decisions in different color channels [8].

Another widespread method is Principal Component Analysis (PCA) that has been run on the grayscale images of same database that we are using and hence we are using it as a tool to compare the performance of the proposed algorithm by us $[9,10]$. There has been various works conducted on iris recognition dealing with iris images in different light spectrum [11].

This paper is organized as follows: in the next section the process of iris segmentation is explained. Afterwards, the proposed algorithm is explained in details followed by a 
section on experimental results. In the end conclusion is represented.

\section{IRIS SEGMENTATION}

The first major step of the recognition workflow is to get only the iris out of the image of an eye. It is vital to leave as much of the patterns of the iris as possible, but on the other hand remove all the disturbing noise, which affects the decision like parts of pupil, eyelids, eyelashes, and reflections of light.

Besides the algorithm introduced by Daugman that is described by eqn. (1) there is also Hough transform, which is very popular in the area [12].

However, as we are presenting an iris recognition algorithm in the current paper and not dealing with filtering itself, we have developed manually a suitable mask that takes into account all the irises in the database. It has to be noted that the mask can't be used on any other database and other mean of filtering has to be used when trying to implement the algorithm with some other irises.

In order to obtain noise free irises that we can start to use in the recognition process, we apply Boolean AND operator between the mask and the filter. Figures 1, 2 and 3 illustrate the process.

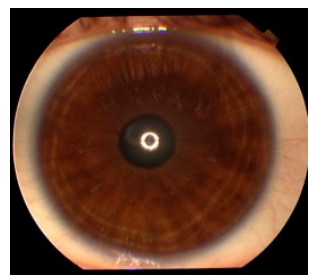

Fig. 1. Original iris with noise

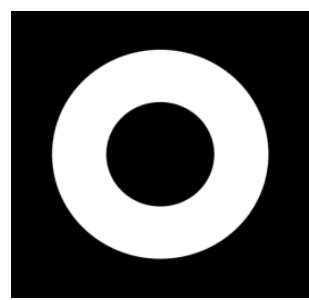

Fig. 2. Manually designed filter

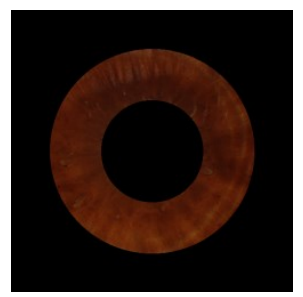

Fig. 3. Iris without noise

\section{Proposed IRIS ReCOGNITION TEChNIQUE}

One of the main questions of iris recognition using texture and color information is what channels to use as a trustworthy source of information and how to combine the data so that we would enhance the recognition rate as much as possible [7].

In order to generate histograms of the filtered images, we need to convert the irises from traditional RGB color space to HSI and YCbCr color space.

RGB color space is not being used because it includes both chrominance and luminance information into the three channels and therefore the color space itself is very sensitive to any kind of changes in lightning conditions and might affect the recognition rate significantly. Instead, both HSI and $\mathrm{YCbCr}$ are used for the identification process. These color spaces are suitable for us as they separate the tonality and illumination information. Additionally channel I has been excluded, because it includes theoretically the same information as $\mathrm{Y}$ and thus we need to remove one of them not to emphasize the influence of the illumination. The number of independent channels and decisions can be increased but in that case we would increase also computational complexity and that is always the unwanted result.

In order to reliably compare the irises, we need to have probability distribution functions (PDF) of all the channels of all the irises. The first step to obtain the PDFs is to generate the corresponding histograms.

A histogram represents the tonal distribution of an image. In simpler terms, it means that we collect and plot the number of specific pixel values and is defined by eqn. (2) and eqn. (3).

$$
\begin{gathered}
H=\left[\varepsilon_{0}, \varepsilon_{2}, \varepsilon_{3}, \ldots, \varepsilon_{255}\right] \\
N=\sum_{i=0}^{255} \varepsilon_{i}
\end{gathered}
$$

In eqn. (2) $\mathrm{N}$ represents the total number of pixels in the image. Probability distribution function (PDF) is a normalized histogram, where the sum of all the elements is always 1 . This means that in order to convert a traditional histogram into PDF, we need to divide all the values in eqn. (2) by $\mathrm{N}$ from eqn. (3). Hence, eqn. (3) will take the form described by eqn. (4).

$$
H=\left[\varepsilon_{0} / N, \varepsilon_{2} / N, \varepsilon_{3} / N, \ldots, \varepsilon_{255} / N\right]
$$

As it can be noticed from eqn. (2)-(4), we will use the bin size of 256, but this can be altered in a way that seems the most convenient. It is important to note that reducing the number of bins means that we will lose precision and this might result in false matches, because in case of less bins different pixel values are considered as being in the same categories. Hence, it could be compared with down sampling an image.

Kullback-Leibler Divergence is a tool to measure the similarity of two irises that we will use in this paper. It is described by eqn. (5).

$$
D_{K L}(P \| Q)=\sum_{i=0}^{255} \ln \left(\frac{P(i)}{Q(i)}\right) * P(i)
$$

$\mathrm{P}(\mathrm{i})$ in eqn. (5) notes the PDF of an iris in the database and Q(i) the input iris. Vidyasagar [13] defines it as relative entropy between two probability density functions $P$ and $Q$.

The more similar the two PDF vectors are to each other, the smaller the value of KLD is, reaching zero for identical vectors and theoretically infinity for vectors that are totally different from each other [13]. All in all, KLD is an ideal tool 
to compare similarity between two irises as the irises of the same eye should have very similar histograms with minor fluctuation caused by different conditions while taking the image.

In order to compare two irises, we need to calculate the corresponding KLD values of $\mathrm{H}, \mathrm{S}, \mathrm{Y}, \mathrm{Cb}$ and $\mathrm{Cr}$ channel by using the PDFs obtained in the previous section. Finally, the similarity of two irises in the context of this paper is found by adding all the KLD values and then dividing the sum by 5 i.e. finding the mean of the calculations.

The method does not use the RGB color space because it encodes both luminance and chrominance information into 3 channels and hence, due to its sensitivity on slight illumination changes affects also the recognition rate.

Combining channel statistics in the mentioned way means that an error in one of the channels will be probably cancelled out by the others. Table 1 shows clearly that recognition rate by using one specific channel is really low and in order to get more precise identification, we need to use the combination of different channels and leave out the ones that will duplicate each other.

The decision making process is described by eqn. (6) where $a_{i}$ is the average KLD value for both left and right irises for channels $\mathrm{H}, \mathrm{S}, \mathrm{Y}, \mathrm{Cb}$ and $\mathrm{Cr}$.

$$
\text { decision }=\min \left(a_{0} \ldots a_{n}\right)
$$

\section{EXPERIMENTAL RESULTS}

The UPOL database [14] is used for obtaining experimental results. It consists of 64 pair of irises classes (left and right) and each class has 3 samples of every iris. Table I is showing the recognition rate of each color channel for the left and right irises separately. The training and test irises have been randomly assigned for each class. In order to have an unbiased performance, the process of recognition with given number of training set has been repeated 100 times and the reported recognition rate is average of the performances.

TABLE I. RECOGNITION RATES OF THE PROPOSED PDF BASED BY CHANNELS (\%)

\begin{tabular}{|c|c|c|c|}
\hline \multirow{2}{*}{ Direction } & \multirow{2}{*}{$\begin{array}{c}\text { Channel } \backslash \text { Number } \\
\text { of training }\end{array}$} & \multicolumn{2}{|c|}{ Recognition rate $(\%)$} \\
\hline & & 1 & 2 \\
\hline \multirow{6}{*}{$\begin{array}{l}\stackrel{\sqcup}{I} \\
\stackrel{\square}{\Xi}\end{array}$} & $\mathrm{H}$ & 64.06 & 78.13 \\
\hline & $\mathrm{S}$ & 75 & 87.5 \\
\hline & $I$ & 46.88 & 65.63 \\
\hline & $\mathrm{Y}$ & 46.88 & 65.63 \\
\hline & $\mathrm{Cb}$ & 62.5 & 78.13 \\
\hline & $\mathrm{Cr}$ & 60.16 & 75 \\
\hline \multirow{6}{*}{ 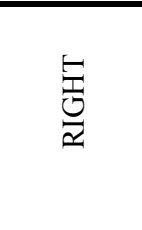 } & $\mathrm{H}$ & 78.13 & 84.38 \\
\hline & $\mathrm{S}$ & 78.13 & 85.94 \\
\hline & $I$ & 60.94 & 73.44 \\
\hline & $\mathrm{Y}$ & 60.94 & 73.44 \\
\hline & $\mathrm{Cb}$ & 65.63 & 78.13 \\
\hline & $\mathrm{Cr}$ & 69.53 & 81.25 \\
\hline
\end{tabular}

As can be seen from Table II, applying mean rule to $\mathrm{H}, \mathrm{S}$, $\mathrm{Y}, \mathrm{Cb}$, and $\mathrm{Cr}$ color channels on just left or right iris for recognition enhances the performance significantly. In order to improve the recognition rate the decision of right and left irises is also combined by using mean rule. This means that instead of 5 channels we will use 10 channels and the probability recognition will be increased. Table III describes this result using the mean rule with both left and right irises. As it can be seen, the algorithm performs significantly better than PCA applied to the grayscale iris images. Also Table III shows the comparison of the proposed technique with a state-of-the-art technique proposed in [8]. Although our proposed technique in case of existence of only 1 sample per class in training set is not over performing, the proposed technique has less computational complexity than [8], as in [8] cross correlation coefficient is used which is a well-known expensive operation. The proposed technique is exclusively suitable for visible light data due to the fact that PDF of color channels in visible light spectrum is being used.

TABLE II. RESULTS USING THE MEAN RULE.BY SEPARATING LEFT AND RIGHT IRISES

\begin{tabular}{|c|c|c|c|}
\hline \multirow{2}{*}{ \# of training } & \multirow{2}{*}{ PCA (\%) } & \multicolumn{2}{|c|}{$\begin{array}{c}\text { Proposed mean rule } \\
\text { based technique (\%) }\end{array}$} \\
\cline { 3 - 4 } & & Left iris & Right iris \\
\hline $\mathbf{1}$ & 60 & 88.3 & 93.8 \\
\hline $\mathbf{2}$ & 70 & 92.2 & 98.4 \\
\hline
\end{tabular}

TABLE III. RESULTS USING THE MEAN RULE AND BOTH LEFT AND RIGHT IRISES

\begin{tabular}{|c|c|c|c|}
\hline \# of training & PCA (\%) & $\begin{array}{c}\text { Histogram based } \\
\text { technique }[\mathbf{8}] \\
(\%)\end{array}$ & $\begin{array}{c}\text { Proposed mean } \\
\text { rule based } \\
\text { technique (\%) }\end{array}$ \\
\hline $\mathbf{1}$ & 60 & 98.91 & 94.5 \\
\hline $\mathbf{2}$ & 70 & 100 & 100 \\
\hline
\end{tabular}

\section{CONCLUSION}

This research work has proposed a new iris recognition technique based probability distribution function of iris images. During the process of identification probability distribution functions of colored irises were generated in HSI and $\mathrm{YCbCr}$ color spaces. KLD was used for discrimination between classes and final decision on recognition was achieved by combination of decision obtained in $\mathrm{H}, \mathrm{S}, \mathrm{Y}, \mathrm{Cb}$, and $\mathrm{Cr}$ color channels by employing mean rule. The experimental results showed that the proposed technique overcame the conventional PCA technique.

\section{ACKNOWLEDGMENT}

This work is partially supported by ERDF program "Estonian higher education information and communications technology and research and development activities state program 2011-2015 (ICT program)" and the European Union through the European Social Fund (MTT76).

\section{REFERENCES}

[1] John G. Daugman, "High confidence visual recognition of persons by a test of statistical independence," Pattern Analysis and Machine Intelligence, IEEE Transactions on, vol. 15, pp. 1148 - 1161, 1993.

[2] Yu Chen; FL Jin Wang ; Changan Han ; Lu Wang ; Adjouadi, M. "A robust segmentation approach to iris recognition based on video," in 
Applied Imagery Pattern Recognition Workshop, 37th IEEE Applied Imagery Pattern Recognition Workshop, pp. 1-8, 2008.

[3] John G. Daugman and C. Downing, "Epigenetic randomness, complexity and singularity of human iris patterns," Proceedings of the Royal Society B, vol. 268, no. 1477, pp. 1737-1740, August 2001.

[4] T. Mansfield, G. Kelly, D. Chandler, and J. Kane, "Biometric Product Testing," National Physical Laboratory, Teddington, 2001.

[5] A. Muroň and J. Pospíšil, "The Human Iris Structure And Its Usages," Palacký University, Olomouc, Olomouc, 2000.

[6] Chia-Te Chou, Sheng-Wen Shih, Wen-Shiung Chen, V.W. Cheng, and Duan-Yu Chen, "Non-Orthogonal View Iris Recognition System," Circuits and Systems for Video Technology, IEEE Transactions on, vol. 20, no. 3, pp. 417-430, March 2010.

[7] F. Melgani, F. De Natale, Chunguang Zhou, Libiao Zhang, and Xiaohua Liu, "Incremental Learning based Color Iris Recognition," in Incremental Learning based Color Iris Recognition, Atizapan de Zaragoza, 2008, pp. 319-324.

[8] H. Demirel and G. Anbarjafari, "Iris recognition system using combined histogram statistics.," in Computer and Information Sciences, 2008. ISCIS '08. 23rd International Symposium on, Istanbul, 2008, pp. 1-4.

[9] Jin-Xin Shi and Xiao-Feng Gu, "The comparison of iris recognition using principal component analysis, independent component analysis and Gabor wavelets," in Computer Science and Information Technology (ICCSIT), 2010 3rd IEEE International Conference on, Chengdu, 2010 pp. 61-64.

[10] J. Shlens, "A Tutorial On Principal Component Analysis," UC San Diego, 2003.

[11] Jiali Cui, Yunhong Wang, Junzhou Huang, Tieniu Tan, and Zhenan Sun, "An iris image synthesis method based on PCA and super-resolution," in Pattern Recognition, 2004. ICPR 2004. Proceedings of the 17th International Conference on, 2004, pp. 471-474.

[12] Yong-zeng Shen, Min-jie Zhang, Jie-wei Yue, and Hong-min Ye, "A New Iris Locating Algorithm," in Artificial Reality and Telexistence-Workshops, 2006. ICAT '06. 16th International Conference on, Hangzhou, 2006, pp. 438 - 441.

[13] M. Vidyasagar, "Divergence, Variational Kullback-Leibler," in Decision and Control, 2007 46th IEEE Conference on, New Orleans, LA, 2007, pp. 6160-6165.

[14] M. Dobeš and L. Machala. (2014, April) Iris Database. [Online]. http://phoenix.inf.upol.cz/iris/ 